\title{
Interaction of FKBP5, a Stress-Related Gene, with Childhood Trauma Increases the Risk for Attempting Suicide
}

\author{
Alec Roy*,', Elena Gorodetsky², Qiaoping Yuan ${ }^{3}$, David Goldman $^{3}$ and Mary-Anne Enoch ${ }^{3}$ \\ 'Psychiatry Service, Department of Veterans Affairs, New Jersey VA Health Care System, East Orange, NJ, USA; ${ }^{2}$ Mood and Anxiety Disorders \\ Program, National Institute of Mental Health, National Institutes of Health, Bethesda, MD, USA; ${ }^{3}$ Laboratory of Neurogenetics, National Institute \\ on Alcohol Abuse and Alcoholism, National Institutes of Health, Bethesda, MD, USA
}

\begin{abstract}
Childhood trauma is associated with hypothalamic-pituitary-adrenal (HPA) axis dysregulation and is a known risk factor for suicidal behavior. In this study we sought to determine whether the impact of childhood trauma on suicide risk might be modified by FKBP5, an HPA-axis regulating gene. Sixteen FKBP5 haplotype-tagging single nucleotide polymorphisms (SNPs) were genotyped in a sample of African Americans: 398 treatment-seeking patients with substance dependence ( $90 \%$ men; 120 suicide attempters) and 432 nonsubstance-dependent individuals (40\% men; 2 I suicide attempters). In all, 474 participants ( I 2 suicide attempters) also completed the Childhood Trauma Questionnaire (CTQ). Primary haplotype analyses were conducted with the four SNPs implicated in earlier studies: rs3800373, rs9296158, rs 1360780, and rs9470080. We found that childhood trauma was associated with suicide attempt $(P<0.000 \mathrm{I})$. Although there was no main effect of the two major yin yang haplotypes in the four SNP haplotype blocks, there was a haplotype influence on suicide risk $(p=0.006)$ only in individuals exposed to high levels of childhood trauma. In this group, 5 I\% with two copies of the risk haplotype, $36 \%$ with one copy, and $20 \%$ with no copies had attempted suicide. The total logistic regression model accounted for $13 \%$ of the variance in attempted suicide. Analyses of the 16 SNPs showed significant main effects on suicide attempt of rs3777747, rs47/3902, and rs9470080 and interactive effects of rs3800373, rs9296158, and rs/360780 with CTQ score on suicide attempt. These data suggest that childhood trauma and variants of the FKBP5 gene may interact to increase the risk for attempting suicide.
\end{abstract}

Neuropsychopharmacology (2010) 35, 1674-1683; doi:I0.1038/npp.2009.236; published online 20 January 2010

Keywords: neurogenetics; addiction and substance abuse; behavioral science; biological psychiatry; suicide

\section{INTRODUCTION}

Suicide is the fifth leading cause of years of potential life lost before age 75 years and the eleventh leading cause of death in the Unites States (Castle et al, 2004). Data from family, twin, adoption, and molecular genetic studies show that there is a genetic component to suicidal behavior (reviewed in Bondy et al, 2006). Exposure to childhood trauma is also recognized as an independent risk factor for suicidal behavior. Childhood trauma is common: a general population survey revealed that $31 \%$ of men and $21 \%$ of women reported a history of childhood physical abuse whereas childhood sexual abuse was reported by $13 \%$ of women and $4 \%$ of men (Holmes and Slap, 1998; MaMillan et al, 1997). Results from the Epidemiologic Catchment Area (ECA)

*Correspondence: Dr A Roy, Psychiatry Service (I I6A), Department of Veterans Affairs, New Jersey VA Health Care System, 385 Tremont Ave, East Orange, NJ 07018, USA, Tel: + I 973676 1000,

Fax: + | 973395 7766, E-mail: Alec.Roy@med.va.gov

Received 4 October 2009; revised 27 November 2009; accepted 18 December 2009 study and the US National Comorbidity Survey have shown a strong association between childhood sexual abuse and suicidal behavior (Davidson et al, 1996; Molnar et al, 2001). Similarly, an Australian community study of twins found that a history of childhood trauma significantly increased the risk of a suicide attempt (Nelson et al, 2002). Moreover, data from the US National Comorbidity Survey Replication indicate that exposure to childhood physical abuse, sexual abuse, or witnessing domestic violence accounts for 16 and $50 \%$ of suicidal ideation and attempts, respectively, among women and 21 and $33 \%$ of ideation and attempts among men (Afifi et al, 2008). Clinical studies have similarly reported that childhood trauma is associated with suicide attempts in patients with various psychiatric disorders (Brodsky and Stanley, 2007; Roy, 2001a, 2001b; Sarchiapone et al, 2007).

Childhood trauma has also been shown to impact stress reactivity in adulthood by altering hypothalamicpituitary-adrenal (HPA) axis function (De Bellis et al, 1994; Heim et al, 2004; Nemeroff, 2004; Roy, 2002a). Similarly, preclinical studies in both primates and rodents show that early life trauma impacts HPA axis function in the offspring 
(Meaney, 2001; Sánchez et al, 2005; Weaver et al, 2005). Moreover, HPA axis dysregulation occurs in suicidal individuals. For example, changes on the dexamethasone suppression test, in $24 \mathrm{~h}$ urinary free cortisol outputs, salivary cortisol, cerebrospinal fluid levels of corticotrophin-releasing hormone $(\mathrm{CRH})$, and $\mathrm{CRH}$ binding sites in frontal cortex have been reported in individuals exhibiting suicidal behavior (Arato et al, 1989; Bunney and Fawcett, 1965; Bunney et al, 1969; Coryell and Schlesser, 2001; Lindqvist et al, 2008; Nemeroff et al, 1988; Pfennig et al, 2005; Roy et al, 1986; Roy, 1992). Furthermore, decreased expression of the glucocorticoid receptor has been detected after postmortem of hippocampus from suicide victims exposed to childhood trauma (McGowan et al, 2009).

The mechanism of HPA axis dysregulation includes dysregulation of the hypothalamic peptides $\mathrm{CRH}$ and arginine vasopressin (AVP) leading to increased release of plasma ACTH and cortisol. This may be coupled with glucocorticoid receptor insensitivity that results in impairment of the negative feedback loop. Glucocorticoid receptor activation and ligand binding are moderated by a large molecular complex that includes FKBP5, a co-chaperone of hsp90. Upon ligand binding the glucocorticoid receptor dissociates from the chaperone complex and migrates to the nucleus where it initiates transcription in the target genes, $C R H$ and $A V P$. Binder et al (2004) genotyped single nucleotide polymorphisms (SNPs) in the glucocorticoid receptor, the target genes, and five co-chaperones in the regulatory complex and detected a relationship between FKBP5 and depression phenotypes. FKBP5 SNPs have been associated with the response to antidepressants, the recurrence of depressive episodes (Binder et al, 2004), suicide attempts in bipolar patients (Willour et al, 2009), and the incomplete normalization of stress-elicited cortisol secretion (Ising et al, 2008). Moreover, Binder et al reported that in an African-American sample, four SNPs (rs3800373, rs9296158, rs1360780, and rs9470080) interacted with childhood trauma in predicting symptoms of posttraumatic stress disorder (PTSD), a disorder associated with both a raised risk of attempting suicide and HPA axis dysregulation (Binder et al, 2008; Wilcox et al, 2009). Therefore, it may be that FKBP5 may moderate the sensitization by childhood trauma of the stress-responsive HPA pathway.

We administered the Childhood Trauma Questionnaire (CTQ) to a large group of African-American participants who had also been interviewed about the history of suicide attempts. Data from the ECA study found a 2.3\% lifetime prevalence of attempted suicide among African Americans and showed that African Americans were as likely to attempt suicide as were other racial-ethnic groups (Moscicki et al, 1998). The majority of our AfricanAmerican suicide attempters were substance-dependent patients, a psychiatric diagnosis associated with childhood trauma and with a raised risk of suicidal behavior (Arnow, 2004; Harris and Barraclough, 1997; Hyman et al, 2006; Johnsson and Fridell 1997; Kosten and Rounsaville 1988; Nelson et al, 2006; Roy, 2001a, 2001b, 2002b, 2003; Roy and Janal, 2007). Because suicidal behavior is associated with childhood trauma and HPA axis dysregulation, we decided to test the hypothesis that the interaction between childhood trauma and the FKBP5 gene might increase the risk for attempting suicide, at least in substance-dependent individuals in whom suicidality is frequently seen. Our primary haplotype analyses were conducted with the four previously implicated FKBP5 SNPs (rs3800373, rs9296158, rs1360780, and rs9470080) but we also performed secondary analyses to determine whether denser SNP coverage of FKBP5 (11 SNP haplotypes) would yield more information.

\section{PARTICIPANTS AND METHODS}

\section{Participants}

The sample consisted of two subsamples of AfricanAmerican participants recruited from the same geographic area that were pooled for this study. The first subsample consisted of 706 African-American patients (634 men, 72 women) recruited from the Substance Abuse Treatment Program (SATP) at the Department of Veteran Affairs New Jersey Health Care System (VANJHCS), East Orange Campus. Nearly all were inpatients on a 21-day residential treatment ward, however, a few were recruited from the SATP outpatient clinic or from the methadone clinic. Criteria for inclusion in the study were that patients were $\geqslant 18$ years of age, met DSM-IV criteria for alcohol, cocaine, or opiate dependence, self-identified as African American, and were abstinent. Exclusion criteria included mental retardation, dementia, and acute psychosis. Patients were interviewed by a psychiatrist (AR) with a semi-structured interview that included both the substance abuse section of the Structured Clinical Interview for DSM-IV (SCID) (Spitzer et al, 1995) to determine lifetime substance dependence diagnoses, and questions about whether the patient had ever attempted suicide. A suicide attempt was defined as a self-destructive act with some intent to end one's life that was not self-mutilatory in nature. The information from the patient about suicide attempts was supplemented by collateral information from mental health program staff, medical records, the program internist and physician's assistant, and, where possible, from treating mental health professionals. The definition of suicide attempt was implemented by a psychiatrist (AR) after interviewing the patient with a standard series of clinical questions and reviewing all of the collateral information. Their mean (SD) age was 45.2 (7.9) years.

The second subsample consists of 759 African-American men $(n=307)$ and women $(n=452)$ recruited from churches and a blood bank in Newark, NJ, (46\%) and from a consecutive series of insulin-dependent diabetic outpatients seen at an ophthalmology clinic (54\%) at the University of Medicine and Dentistry, New Jersey Medical School (UMDNJ), Newark, NJ. These participants had a semi-structured psychiatric interview conducted by either a physician or a research worker (reviewed by AR) that included a standard series of questions about lifetime suicidal behavior and substance abuse/dependence. Their mean (SD) age was 34.3 (10.1) years. These individuals were initially recruited as potential controls for genetic studies in substance dependence.

After a full description of the study was provided, all participants gave written informed consent to the study that was approved by the institutional review boards of the VANJHCS and UMDNJ. 


\section{Childhood Trauma Questionnaire}

The CTQ (28-item version) was completed by 538 patients ( $92 \%$ men) with substance dependence; 166 (90\% men) had attempted suicide. The CTQ was completed by 342 nonsubstance-dependent participants ( $42 \%$ men), 32 (28\% men) of whom had attempted suicide (see Figure 1).

The CTQ yields scores for five traumas experienced in childhood: physical abuse, physical neglect, emotional abuse, emotional neglect, and sexual abuse, as well as a total score. Reliability and validity of the CTQ have been shown, including in drug abusers and African-American populations (Bernstein et al, 1997, 1994, 2003; Bernstein and Fink, 1998; Scher et al, 2001; Thombs et al, 2007). Some of the patients' CTQ data have been previously reported (Roy, 2001a, 2001b, 2002b, 2003). CTQ scores for each of the five traumas range from 5 to 25 . The total CTQ score ranges from 25 to 125 . The CTQ was used as a continuous measure in all logistic regression analyses.

A dichotomous total CTQ score was derived for use in secondary analyses to illustrate the direction of interaction effects on suicide attempt. A total CTQ score $\geqslant 1$ SD above the mean CTQ score of nonsubstance-dependent/no suicide attempt participants $(36.49$ (11.67), ie, $\geqslant 48)$ was designated 'high level of childhood trauma' $(N=290)$; lower total CTQ scores $(<48)$ were designated 'low level of childhood trauma' $(N=590)$.

\section{Genotyping}

Genomic DNA was isolated from blood of 830 participants using standard protocols. Sixteen haplotype-tagging SNPs spanning FKBP5 were selected; rs numbers for the 16 SNPs, the bases for alleles $1-2$, together with the allele 2 frequencies, are shown in Table 1. Alleles 1 and 2 of each SNP are located on opposite DNA strands. All SNPs were genotyped using a TaqMan Assay-on-Demand (Applied Biosystems, Foster City, CA). Genotyping was performed according to the manufacturer's protocol and genotype was determined at end point using an ABI 7900HT Sequence Detection System. Genotyping accuracy was determined empirically by duplicate genotyping of $25 \%$ of the samples selected randomly. The error rate was $<0.005$, and the completion rate was $>0.95$. All SNPs were in HardyWeinberg equilibrium $(p>0.14)$ with the exception of rs9470067 $(p=0.001)$, which was subsequently excluded from all analyses.

\section{Summary of Data Set}

The data set is summarized in Figure 1. Both genotype and CTQ data were available for 474 individuals: 112 suicide attempters (224 chromosomes for haplotype analyses) and 362 nonsuicide attempters ( 724 chromosomes for haplotype analyses). Missing DNA and CTQ data were random and showed no selection bias. Supporting evidence for this can be seen in Figure 1: the sex ratio was the same in the total

Table I Description of FKBP5 SNPS

\begin{tabular}{|c|c|c|c|c|}
\hline \multirow{2}{*}{$\begin{array}{l}\text { FKBP5 } \\
\text { SNPs }\end{array}$} & \multicolumn{2}{|c|}{ Location } & \multirow{2}{*}{$\begin{array}{c}\text { Base } \\
\text { variation } \\
\text { Alleles I-2 }\end{array}$} & \multirow{2}{*}{$\begin{array}{c}\text { freq } \\
\text { Allele } 2\end{array}$} \\
\hline & chr 6 & Gene & & \\
\hline rs3800373 & 35650454 & $3^{\prime}$ UTR & C-A & 0.60 \\
\hline rs9470065 & $35658|3|$ & Intron 8 & G-A & 0.08 \\
\hline rs9296I58 & 35675060 & Intron 5 & G-A & 0.45 \\
\hline rs47।3899 & 35677259 & Intron 5 & G-A & 0.10 \\
\hline rs9470067 & 35684834 & Intron 5 & G-A & 0.09 \\
\hline rs3777747 & 35686980 & Intron 5 & G-A & 0.61 \\
\hline rs7762668 b & 35687500 & Intron 5 & G-A & 0.01 \\
\hline rs9462099b & 35688782 & Intron 5 & $\mathrm{~T}-\mathrm{C}$ & 0.04 \\
\hline rs9380524b & 35697048 & Intron 3 & C-A & 0.02 \\
\hline rs 1360780 & $357 \mid 5549$ & Intron 2 & $\mathrm{~T}-\mathrm{C}$ & 0.61 \\
\hline rs $7771722^{b}$ & 35721976 & Intron I & G-A & 0.09 \\
\hline rs47/3902 & 35722004 & Intron I & $\mathrm{T}-\mathrm{C}$ & 0.09 \\
\hline rs9462100 & 35723690 & Intron I & $\mathrm{T}-\mathrm{C}$ & 0.12 \\
\hline rs2092427 & 35730185 & Intron I & G-A & 0.22 \\
\hline rs7762760 & 35745319 & Intron I & G-A & 0.02 \\
\hline rs9470080 & $357544 \mid 3$ & Intron I & $\mathrm{T}-\mathrm{C}$ & 0.56 \\
\hline
\end{tabular}

SNPs in bold were included in the 4 SNP haplotype analyses.

${ }^{a}$ Rs9470067 genotypes were not in Hardy-Weinberg equilibrium and this SNP was therefore excluded from analyses.

${ }^{\mathrm{b}}$ These 4 SNPs were not included in the II SNP haplotype analyses since they were not in LD with the other SNPs.

\section{SUBSTANCE DEPENDENCE}

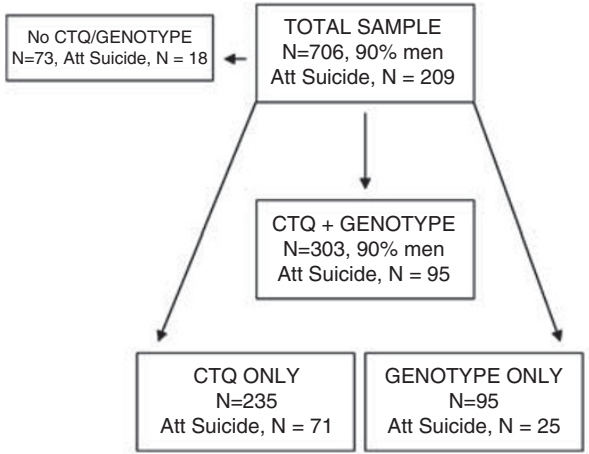

NO SUBSTANCE DEPENDENCE

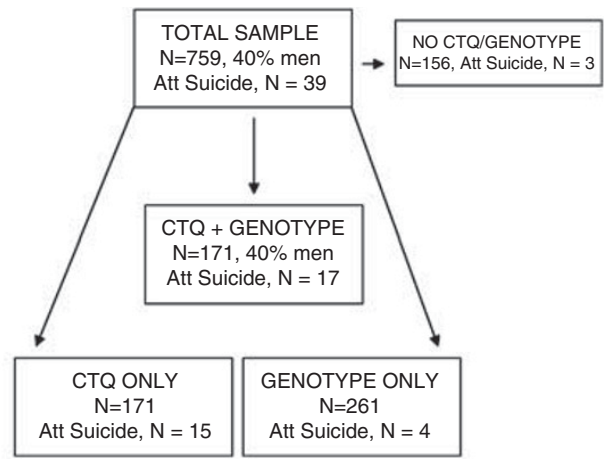

Figure I Summary of data set. 
group of substance-dependent individuals and the subgroup with both CTQ and genotype data (both 90\% men); likewise for the nonsubstance-dependent individuals (both groups had $40 \%$ men). Moreover, the proportion of suicide attempters was the same in all four subgroups of substancedependent individuals shown in Figure $1(p=0.61)$.

\section{Assessment of Population Stratification Using Ancestry Informative Markers}

The samples were genotyped for 186 ancestry informative markers (AIMs) (Hodgkinson et al, 2008). The same AIMs were also genotyped in 1051 individuals from the 51 worldwide populations represented in the HGDP-CEPH Human Genome Diversity Cell Line Panel (http://www. cephb.fr/HGDP-CEPH-Panel). Structure 2.2 (http://pritch. bsd.uchicago.edu/software.html) was run simultaneously using the AIMs genotypes from our sample and the 51 $\mathrm{CEPH}$ populations to identify population substructure and compute individual ethnic factor scores. This ancestry assessment identifies seven ethnic factors that have been previously described (Stephens and Donnelly, 2003). In this sample of African Americans, the mean African factor score was 0.77 (median $=0.81$ ) and the mean European factor score was 0.09 (median value $=0.04$ ). Both a Mid East factor and an Asian factor had an average score of 0.06 (median 0.04).

\section{Statistical Analyses}

The primary analyses in this study were haplotype analyses conducted with the four SNPs: rs3800373, rs9296158, rs1360780, and rs 9470080 that span $130 \mathrm{~kb}$ across FKBP5, are in strong linkage disequilibrium (LD) (Figure 2), and have consistently featured significant results in earlier publications (Binder et al, 2004, 2008; Ising et al, 2008; Willour et al, 2009). Secondary analyses were performed to elucidate the results of the primary analyses: (1) to determine whether denser SNP coverage (11 SNP haplotypes)

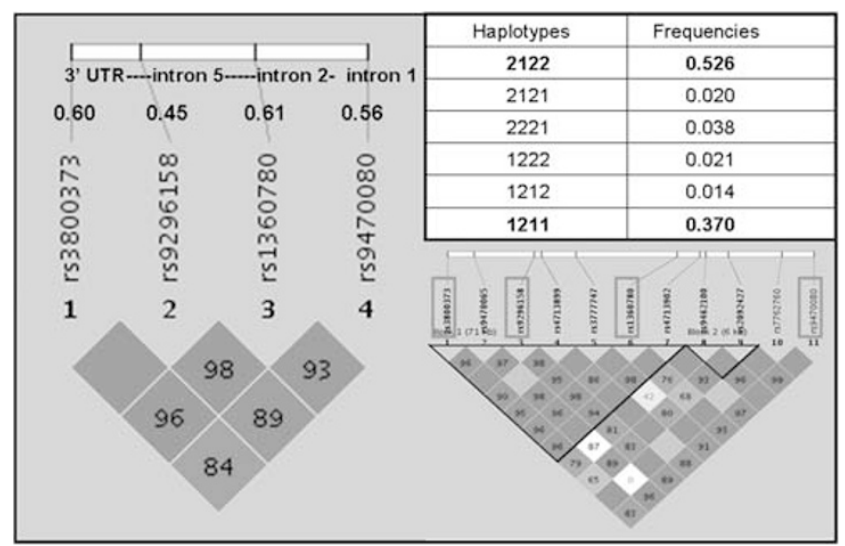

Figure 2 FKBP5 II single nucleotide polymorphism (SNP) haplotype block structure and four SNP haplotype block and haplotypes. The numbers in the squares refer to pair-wise linkage disequilibrium (LD) measured as $D^{\prime}$. Haplotype blocks were defined using a setting of average pair-wise $D^{\prime}$ within-block of $\geqslant 0.80$. The left panel shows the haplotype block structure for the four SNPs highlighted in the right panel; the four SNP haplotypes are listed and the yin yang haplotypes are indicated in bold. would yield more information, (2) analyses to illustrate the direction of interaction effects on suicide attempt, and (3) individual SNP analyses to determine whether any of the SNPs provided the signals for the haplotype analyses. Therefore the $p$-values for the secondary analyses were not adjusted for multiple comparisons. Nevertheless it should be noted that the whole model results for the secondary haplotype analysis $(p<0.0001)$ and the secondary six nonindependent SNP analyses (all $p<0.0001$ ) were all highly significant.

Logistic regression analyses were undertaken using JMP 7 software. In the primary analysis, the yin yang haplotypes 2122 and 1211 were included as dependent variables in the model (Table 2) and in secondary analyses, the 11 SNP haplotypes $\mathrm{H} 2$ and $\mathrm{H} 5$ were included as dependent variables (Table 3). Backward stepwise regression was performed with variables being eliminated from the model in an iterative process. The continuous CTQ score and, ethnic factor scores, and the dichotomous variables: substance dependence diagnosis, age, and sex were included as covariates in the final model if they had significant effects. The interaction term was included in the final model when significant. Logistic regression models with nominal variables yielded likelihood ratio (L-R) $\chi^{2}$ results.

Logistic regression results for four SNPs (rs9470065, rs4713899, rs7771722, and rs4713902) were unstable due to the low numbers of the 22 homozygotes, therefore analyses were performed on the combined 22 and 12 genotypes.

Haplotype frequencies were estimated using a Bayesian approach implemented with PHASE (Stephens and Donnelly, 2003). Haploview version 2.04 software (Whitehead Institute for Biomedical Research, USA) was used to produce LD matrices. Because rare and uncommon haplotypes are subject to estimation errors because of increased sampling variance, all analyses were conducted with haplotypes $\geqslant 5 \%$ frequency.

Although the mean (SD) age of substance-dependent patients (45.2 (7.9)) was higher than nonsubstance-dependent participants (34.3 (10.1), $\mathrm{F}=211, p<0.0001)$ there was no correlation between age and CTQ score in either patients

Table 2 The Influence of the Primary Four SNP Yin Yang Haplotypes and Childhood Trauma on Attempted Suicide

\begin{tabular}{lcc}
\hline Parameters & L-R tests $\chi^{\mathbf{2}}$ & $\boldsymbol{P}$-value \\
\hline FKBP5 yin yang haplotypes: 2122, 1211 & 2.63 & 0.11 \\
European ethnic factor score & 7.59 & 0.006 \\
Substance Dependence Diagnosis & 31.42 & $<0.0001$ \\
Total CTQ score & 27.63 & $<0.0001$ \\
G $\times$ E & 4.53 & 0.006 \\
\hline
\end{tabular}

Whole model test: $\chi^{2}=111 ;$ df $=5 ; p<0.0001, r^{2}=0.13$.

The effect likelihood ratio tests (L-R) are shown (I df for each test).

The yin yang haplotypes account for $90 \%$ of total haplotype diversity (see Figure 2).

$N=749$. Since these are haplotype analyses the $N$ represents the number of chromosomes (2 per individual).

The total Childhood Trauma Questionnaire (CTQ) score is a continuous measure.

$\mathrm{G} \times \mathrm{E}$ indicates the interaction between yin yang haplotypes and total

CTQ score. 
Table 3 The Influence of Two II SNP Haplotypes, $\mathrm{H} 2$ and $\mathrm{H} 5$, and Childhood Trauma on Attempted Suicide

\begin{tabular}{lcc}
\hline Parameters & L-R tests $\boldsymbol{\chi}^{\mathbf{2}}$ & $\boldsymbol{p}$-value \\
\hline I I SNP haplotypes H2, H5 & 1.38 & 0.239 \\
European ethnic factor score & 5.20 & 0.023 \\
Substance Dependence Diagnosis & 17.24 & $<0.000$ I \\
Total CTQ score & 6.83 & 0.009 \\
G $\times$ E & 6.89 & 0.009 \\
\hline
\end{tabular}

Whole model test: $\chi^{2}=43 ; \mathrm{df}=5 ; p<0.0001, r^{2}=0.19$.

The effect likelihood ratio tests (L-R) are shown ( I df for each test).

$N=230$. As these are haplotype analyses, the $N$ represents the number of

chromosomes (2 per individual). Only carriers of haplotypes $\mathrm{H} 2$ (freq $=17 \%$ ) and $\mathrm{H} 5$ (freq $=9 \%$ ) were included in these analyses. Haplotype $\mathrm{H} 2$ :

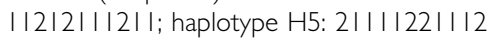

The total childhood trauma questionnaire (CTQ) score is a continuous measure. $\mathrm{G} \times \mathrm{E}$ indicates the interaction between haplotypes $\mathrm{H} 2, \mathrm{H} 5$, and total $\mathrm{CTQ}$ score.

( $r=0.07, p=0.211)$ or nondependent individuals $(r=0.09$, $p=0.276$ ). Inclusion of age as a covariate had no effect on outcomes.

There were no sex differences in CTQ scores within the categories: nonsubstance-dependent/no suicide attempt, substance-dependent/no suicide attempt, nonsubstancedependent + suicide attempt, substance-dependent + suicide attempt. Therefore both sexes were included together in the analyses.

\section{RESULTS}

Substance abuse was associated with significantly higher total CTQ scores $(\mathrm{F}(1,879)=100, p<0.0001)$. However, individuals who had attempted suicide had higher CTQ scores irrespective of whether they were substance dependent - mean (SD) CTQ scores in: nonsubstance-dependent/ no suicide attempt $=36.5 \quad(11.7) \quad(n=310)$; substancedependent/no suicide attempt $=46.6(15.2)(n=372)$; nonsubstance-dependent + suicide attempt $=53.0 \quad(18.3) \quad(n=$ 32 ); substance-dependent + suicide attempt $=53.7 \quad$ (19.5) $(n=166), \mathrm{F}(3,876)=54.3, p<0.0001$.

In this sample of African Americans, European admixture significantly influenced the risk of suicide attempt (Tables 2 and 3 ).

\section{Four SNP Haplotype Analyses}

The primary analyses were conducted with the four SNPs (rs3800373, rs9296158, rs1360780, and rs9470080) that have been implicated in earlier studies and which were the subject of our hypothesis. Figure 2 shows that these four SNPs that span FKBP5 are in strong LD. Within this haplotype block there are six haplotypes with $\geqslant 1 \%$ frequency that account for $99 \%$ of the haplotype diversity (Figure 2). There are two major yin yang haplotypes, 2122 and 1211, that account for $90 \%$ of the haplotype diversity. For simplicity, analyses were performed with these two yin yang haplotypes.

Logistic regression analyses were performed to determine the effects of the yin yang haplotypes and childhood trauma

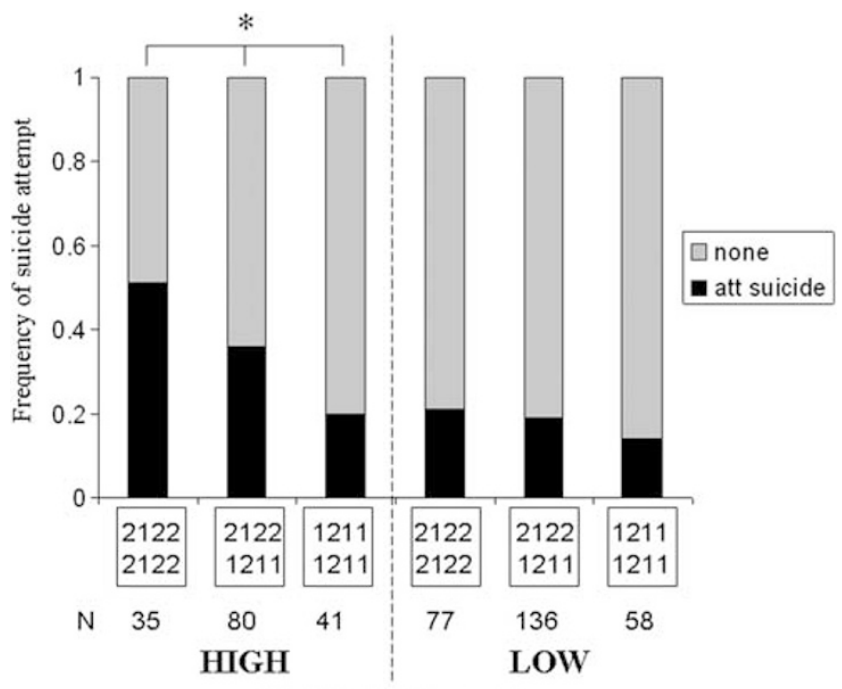

Childhood Trauma

Figure 3 The interaction of FKBP5 yin yang diplotypes with high levels of childhood trauma predicts suicide attempts. High level of childhood trauma = total Childhood Trauma Questionnaire (CTQ) score $\geqslant 1$ SD $(\geqslant 48)$ above mean of nonsubstance-dependent/no suicide attempt participants. Low level of childhood trauma $=$ total CTQ score $<48$. $N=427 ; * \chi^{2}=8.5, \mathrm{df}=2, p<0.014$.

(the continuous total CTQ score) on suicide attempt and the results are presented in Table 2 . Childhood trauma had a significant effect on suicide attempt but there was no main effect of the yin yang haplotypes. However the interaction between the yin yang haplotypes and CTQ score significantly influenced the risk of attempted suicide. The total model shown in Table 2 was significant $\left(\chi^{2}=111, \mathrm{df}=5\right.$, $p=<0.0001)$ and contributed to $13 \%$ of the variance in attempted suicide.

\section{Secondary Analyses}

\section{Interaction Between CTQ Score and Four SNP Diplotypes}

To illustrate the direction of the interaction effect on suicide attempt, we used the previously described dichotomous CTQ high/low variable (high level of childhood trauma designated as CTQ total score $\geqslant 1$ SD above the mean score of nonsubstance-dependent/no suicide attempt participants; lower CTQ scores were designated low level of childhood trauma). Figure 3 shows the individuals who had experienced a high level of childhood trauma, $51 \%$ of those with two copies of the 2122 haplotype had attempted suicide compared with $36 \%$ of those with one copy of 2122 and $20 \%$ of those with no copies of the risk haplotype $\left(\chi^{2}=8.5\right.$, $\mathrm{df}=2, p=0.014)$. In contrast, haplotype 2122 conferred no risk to the individuals who had been exposed to low levels of childhood trauma. Similar results were found if the same diplotype analysis was performed only in the substancedependent participants $(N=274): 56 \%$ of those with two copies of the 2122 haplotype had attempted suicide compared with $39 \%$ of those with one copy of 2122 and $23 \%$ of those with no copies of the risk haplotype $\left(\chi^{2}=7.0\right.$, $\mathrm{df}=2, p=0.031$ ) whereas genotype had no effect on individuals who had been exposed to low levels of childhood trauma (26-30\% suicide attempters). 


\section{Eleven SNP Haplotype Analyses}

Further secondary analyses were performed to determine whether denser SNP coverage would yield more information about haplotype-diplotype associations. There is strong LD across the whole gene within which we had genotyped 16 SNPs (Table 1), however 4 low-frequency SNPs clustered in the middle of the haplotype block (rs7762668, rs9462099, rs9380524, and rs7771722) showed poor LD and were eliminated from the haplotype analyses. Moreover, as rs9470067 genotypes were not in Hardy-Weinberg equilibrium this SNP was also excluded from the analyses.

The resulting 11 SNP haplotype analyses indicated one haplotype block across the gene (Figure 2). There were 14 haplotypes with $\geqslant 1 \%$ frequency that accounted for $95 \%$ of haplotype diversity and 8 haplotypes with $\geqslant 5 \%$ frequency that accounted for $85 \%$ of haplotype diversity. For simplicity, and as rare and uncommon haplotypes are subject to estimation errors because of increased sampling variance, analyses were performed with the haplotypes with $\geqslant 5 \%$ frequency in the total sample: $\mathrm{H} 1,11 \% ; \mathrm{H} 2,17 \%$; $\mathrm{H} 3,9 \%$; $\mathrm{H} 4,18 \%$; $\mathrm{H} 5,9 \%$; H6, 7\%; H7, 6\%; and H8, 8\%. Haplotypes derive from two cladistic groupings: $\mathrm{H} 1-\mathrm{H} 3$ and $\mathrm{H} 4-\mathrm{H} 8$ (Figure 4).

Haplotype H5 was more abundant in those individuals who attempted suicide whereas haplotype $\mathrm{H} 2$ was more abundant in individuals who had not attempted suicide (Figure 4). Moreover, $60 \%$ of individuals with the $\mathrm{H} 5 / \mathrm{H} 5$ diplotype $(N=5)$ had attempted suicide compared with $20 \%$ with the $\mathrm{H} 2 / \mathrm{H} 5$ diplotype $(N=20)$ and $10 \%$ with the $\mathrm{H} 2 / \mathrm{H} 2$ diplotype $(N=30)$. However, the logistic regression analysis (Table 3) revealed no main effect of $\mathrm{H} 2$ or $\mathrm{H} 5$ haplotype on suicide attempt (Table 3 ). Instead, a $\mathrm{G} \times \mathrm{E}$ interaction was apparent. The total model shown in Table 3 was significant $\left(\chi^{2}=43, \mathrm{df}=5, p<0.0001\right)$ and contributed to $19 \%$ of the variance in attempted suicide.

\section{Interaction Between CTQ Score and 11 SNP Haplotypes}

To illustrate the direction of the interaction effect on suicide attempt, we used the previously described CTQ high/low levels of childhood trauma variable. Diplotype analyses

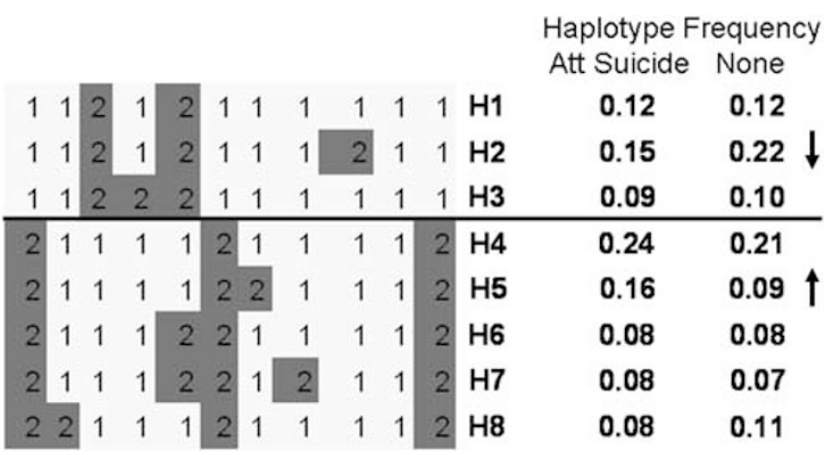

Figure 4 Frequencies of FKBP5 haplotypes in individuals who have attempted suicide compared with nonsuicide attempters. Total number of haplotypes $=1312$. Cladistic grouping of haplotypes $(\mathrm{HI}-\mathrm{H} 3$ and $\mathrm{H} 4-\mathrm{H} 8)$ with frequencies $\geqslant 0.05$ in total sample. Increased frequency of haplotype $\mathrm{H} 5$ and decreased frequency of haplotype $\mathrm{H} 2$ in suicide attempters. SNPs extend left to right from rs3800373 to rs9470080 (see Table I). For each SNP, alleles I and 2 are located on opposite DNA strands. were not possible because, as can be deduced from Figure 4, the frequencies of the 11 SNP diplotypes were too low. Figure 5 shows the individuals in the total group who had experienced a high level of childhood trauma, $62 \%$ of those with the $\mathrm{H} 5$ haplotype had attempted suicide compared with $25 \%$ of those with the $\mathrm{H} 2$ haplotype $\left(\chi^{2}=11,2, \mathrm{df}=1\right.$, $p=0.0008)$. In contrast, haplotype $\mathrm{H} 5$ conferred no risk to the individuals who had been exposed to low levels of childhood trauma.

\section{Individual SNP Analyses}

Secondary analyses were performed with the individual SNPs to determine whether any of the SNPs provided the signals for the findings of the haplotype analyses. Results for the SNPs that were associated with an effect on suicide attempt are summarized in Table 4 . When all significant covariates were included in the logistic regression analyses, the following three SNPs showed significant associations with attempted suicide: rs3777747 located in intron 5 (allele 1 frequency increased in attempted suicide: 0.45 vs 0.38 ); rs4713902 located in intron 1 (allele 2 frequency increased in suicide attempters: 0.14 vs 0.08 ); and rs9470080 located in intron 1 (allele 1 frequency decreased in attempted suicide: 0.38 vs 0.45). Three SNPs showed a $\mathrm{G} \times \mathrm{E}$ interaction: rs3800373, rs9296158, and 1360780 (Table 4). Results with all other SNPs were negative.

From Figure 4 and Table 1 it can be deduced that haplotypes $\mathrm{H} 2$ and $\mathrm{H} 5$ differ in the four SNPs included in the primary haplotype analyses and they also differ in SNPs rs3777747, rs4713902 (unique to H5), and rs2092447 (unique to $\mathrm{H} 2$ ). rs3777747 had a main effect on suicide attempt but its variation is common to several haplotypes. rs2092447 is unique to $\mathrm{H} 2$ but showed no association with

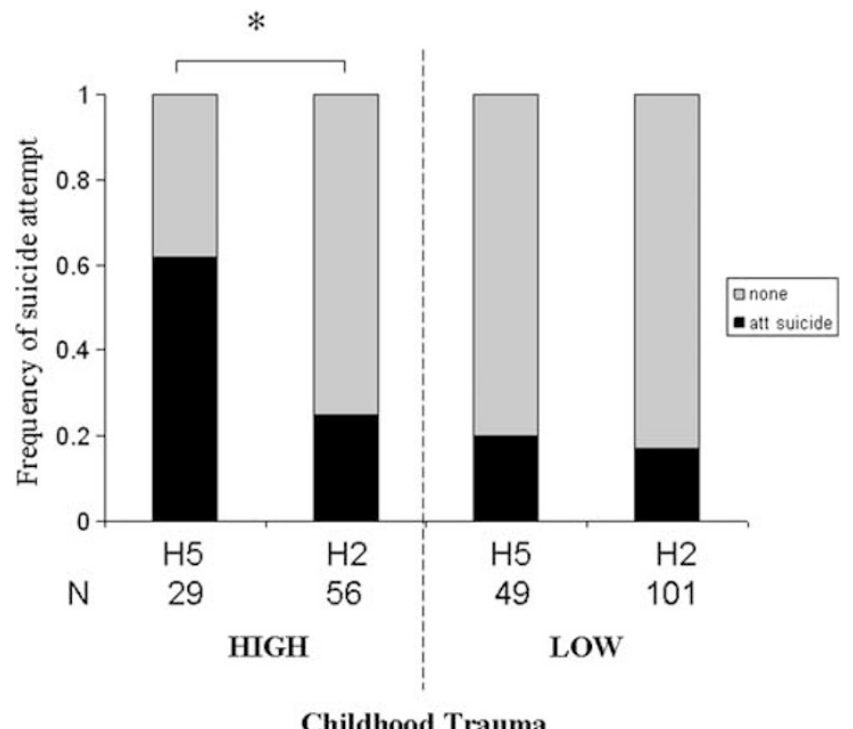

Figure 5 Interaction between FKBP5 haplotypes and childhood trauma; influence on suicide attempt. Haplotype $\mathrm{H} 2$ ( $1|2| 2|1| 2 \mid 1)$, freq $=17 \%$; haplotype $\mathrm{H} 5$ (2I|||22|||2), freq $=9 \%$. High level of childhood trauma = total Childhood Trauma Questionnaire (CTQ) score $\geqslant 1$ SD $(\geqslant 48)$ above mean of nonsubstance-dependent/no suicide attempt participants. Low level of childhood trauma $=$ total $C T Q$ score $<48$. $N=235 ;{ }^{*} \chi^{2}=11.2, \mathrm{df}=1, p=0.0008$. 
Table 4 The Influence of Selected FKBP5 SNPs and Childhood Trauma on Attempted Suicide

\begin{tabular}{|c|c|c|c|c|c|c|c|c|c|c|c|}
\hline SNPs & L-R $\chi^{2}$ & $P$ & $\mathbf{L}-\mathbf{R} \chi^{2}$ & $P$ & L-R $\chi^{2}$ & $P$ & L-R $\chi^{2}$ & $P$ & $\begin{array}{c}\text { Whole } \\
\text { P }\end{array}$ & df & Var \\
\hline Rs3800373 & & & 21.6 & $<0.0001$ & 7.3 & 0.007 & 9.0 & 0.011 & $<0.0001$ & 6 & 0.12 \\
\hline Rs9296158 & & & 18.8 & $<0.0001$ & 11.1 & 0.0009 & 7.2 & 0.028 & $<0.0001$ & 6 & 0.12 \\
\hline Rs3777747 & 7.9 & 0.019 & 17.9 & $<0.0001$ & 21.5 & $<0.0001$ & - & & $<0.0001$ & 4 & 0.11 \\
\hline Rs9470080 & 7.2 & 0.027 & 19.2 & $<0.0001$ & 21.3 & $<0.0001$ & - & & $<0.0001$ & 4 & 0.11 \\
\hline
\end{tabular}

Var, variance explained by total model; $\mathrm{df}$, degrees of freedom.

Results are for effect likelihood ratio (L-R) tests and are given for $p<0.05$.

The childhood trauma effect derives from the childhood trauma questionnaire (CTQ) score, a continuous measure.

df for rs47I3902 is 3 as the II and 12 genotypes were combined to increase power.

All other SNPs showed no significant main or interactive effects on attempted suicide.

attempted suicide. In contrast, rs4713902 is unique to $\mathrm{H} 5$ and showed a significant association with attempted suicide. This suggests that rs4713902 is initiating the 11 SNP haplotypes' association with attempted suicide.

\section{FKBP5 SNP Variation and Substance Dependence}

Logistic regression analyses were performed to determine whether there was any association between any of the SNPs listed in Table 4 (that showed a main or interactive effect on suicide attempt) and substance dependence. The continuous CTQ score and the dichotomous suicide attempt variable (yes or no) were included as covariates as they had significant effects on substance dependence. There were no main gene effects nor was there an interactive effect between FKBP5 SNPs and the continuous CTQ score on substance dependence.

There were no interactive effects between substance dependence, CTQ scores and FKBP5 SNPs on suicide attempt.

Bioinformatics SNP analysis. A bioinformatics search was performed to look at the six SNPs that had either a main or $\mathrm{G} \times \mathrm{E}$ effect on suicide attempt. The search was for potential function such as protein effect by nonsynonymous variants, regulatory effects such as changes in transcription factor binding sites, microRNA sites, or microRNA targets, nearby splice sites or effects on exonic splicing enhancers (ESEs). The only finding was that $\mathrm{rs} 3800373 \mathrm{C}>\mathrm{A}$ causes the loss of the ESE SC35. Although there are some alternative splicing isoforms for FKBP5, rs3800373 is not close to these alternatively spliced exons so it is not known whether this ESE has any importance in this context.

\section{DISCUSSION}

This study has shown that individuals who attempted suicide had experienced significantly greater childhood trauma than individuals who had never attempted suicide. This was the case in both the total group and the substancedependent group and is in accord with previous reports showing that childhood trauma is a risk factor predisposing individuals to attempt suicide when stressed (reviewed in Brodsky and Stanley, 2007). The novel finding from this study comes from the results suggesting that interaction between childhood trauma and the stress-related gene FKBP5 may have a role in suicidal behavior.

In testing our primary hypothesis of interaction with childhood trauma we examined FKPB5 haplotypes derived from the four SNPs that had been implicated in earlier studies (Binder et al, 2004, 2008; Ising et al, 2008; Willour et al, 2009). These four SNPs are in strong LD with each other and also the other seven SNPs that were tested. Because studies have not yet shown which (if any) of the linked SNPs in the haplotype block is functional, it was more appropriate that the primary focus of our study was haplotype analyses. There was no direct effect of the two major yin yang (2122 and 1211) haplotypes on suicidal behavior. However, the interaction between FKPP5 haplotypes and childhood trauma significantly increased the risk of attempting suicide. Among subjects who had experienced high childhood adversity, $51 \%$ of those with two copies of the 2122 haplotype had attempted suicide compared with $36 \%$ of those with one copy and only $20 \%$ of those with no copies. In contrast, there was no gene effect in individuals who had been exposed to little or no childhood trauma. The total logistic regression model accounted for $13 \%$ of the variance in attempting suicide.

Similarly, in a more comprehensive secondary haplotype analysis with 11 SNPs spanning FKBP5 we also detected a significant haplotype-childhood trauma interaction associated with a raised risk of attempting suicide. The total model resulting from this more refined analysis now accounted for $19 \%$ of the variance for attempting suicide. The 11 SNPs were all in strong LD, however, individual SNP analyses revealed a gene $\times$ childhood trauma interactions for rs3800373, rs9296158, and rs1360780 (Table 4). Three SNPs had main effects on suicide attempts: rs9470080, rs3777747, and rs4713902. The latter is of interest as it is unique to the 11 SNP haplotype that was associated with suicide attempt. However, the results of this analysis should be interpreted with caution until replicated due to the small sample size. 
FKBP5 is known to be an inhibitor of glucocorticoid sensitivity therefore elevated levels of FKBP5 might result in the impairment of the HPA axis negative feedback loop.

The SNPs rs3800373, rs9296158, rs1360780, and rs 9470080 are in strong LD and their frequencies are in approximate allelic identity in African Americans (0.39-0.45), suggesting that they may have been subjected to selective pressure. There is some evidence that one or other of these SNPs, or another linked SNP, are functional. Binder et al (2004) found that the minor homozygotes of rs1360780 and rs3800373 were associated with FKBP5 levels in lymphocytes that were twice as high as those with the other two genotypes, however there was no difference in mRNA levels or in plasma cortisol levels. The minor homozygotes of rs1360780 and rs3800373 were associated with a stronger induction of FKBP5 mRNA by cortisol. Further studies are clearly warranted to determine the possible effects of FKBP5 genetic variation on the HPA axis. In our study we found that the major alleles of these four SNPs, together with the minor allele of rs4713902, the SNP that was promoting the 11 SNP haplotype association, were associated with suicide attempt. Moreover, a bioinformatics search revealed that rs3800373 C > A causes the loss of the ESE SC35. Although it is not known whether this ESE has any relevance in vivo, nevertheless in our study the rs3800373 A allele was associated with risk for suicide attempt.

As can be deduced from Table 1 and Figure 3, we found that the more abundant alleles of the four primary SNPs (rs3800373, rs9296158, rs1360780, and rs9470080) were risk factors for suicide attempt in individuals exposed to high childhood adversity. In contrast, Binder et al (2008) using the child abuse section of the Traumatic Events Inventory found that the less abundant variants of these four SNPs were significantly associated with greater PTSD severity in the small group of individuals (approximately 47) exposed to two types of child abuse but not in the larger group exposed to one type of abuse. Although Binder et al (2008) used the CTQ total score in secondary analyses, no results were reported for CTQ interactive effects in the primary analyses with these four SNPs. Because different CTQs were used to detect effects on different outcomes, it is premature to comment at this time on the different direction of FKBP5 SNP associations in our study and that by Binder et al (2008). However, it is tempting to speculate that these differences may be due to contrasting HPA axis sensitivities in PTSD and addiction. Results from studies in PTSD implicate a sensitization of the HPA axis to exposure to stressors (Heim and Nemeroff, 2009; Binder, 2009). In contrast, alcohol and drug dependence are associated with a blunted HPA axis response to stress (Richardson et al, 2008). The addicted participants in this study had such severe substance dependence that they were in an inpatient treatment program and therefore it is likely that their HPA axis responsivity was compromised. Clearly, replication studies in other populations with addiction are needed.

Suicide is generally considered to be a multidetermined act with distal and proximal risk factors. In the stressdiathesis risk factor model of suicidal behavior, both childhood trauma and genetic factors are distal suicide risk factors. They are thought to have their effect by predisposing an individual to an increased risk of attempting suicide when he/she experiences more proximal risk factors such as recent life stress. The fact that a gene implicated in the HPA axis stress-reactivity pathway may be involved in suicidal behavior is of interest given that stress often precipitates suicide attempts. For example, Paykel et al (1975) first showed that those who attempted suicide reported four times as many life events in the 6 months before the attempt than controls. The authors' conclusion that there is strong and immediate relationship between suicide attempts and recent life stress has been replicated in different countries and age groups as well as in completed suicides (Beatrais et al, 1997; Crane et al, 2007; Heikkinen et al, 2007; Osvath et al, 2004). The results of the present study suggest that distal genetic and developmental risk factors may themselves interact to raise the risk of an individual attempting suicide when stressed. In fact in an earlier study we similarly showed that the stress-related serotonin transporter promoter polymorphism (5-HTTLPR) interacted with childhood trauma to predict suicidal behavior in these same substance-dependent patients (Roy et al, 2007). In this study it was the distal risk factors of childhood trauma and the FKBP5 gene that together influenced suicide attempt.

Is there a possible explanation in terms of specific etiologic pathways by which the interaction of FKBP5 and childhood trauma found in the present study might increase the risk of an individual attempting suicide? As FKBP5 impacts glucocorticoid sensitivity, one might speculate that one mechanism underlying this interaction might be the epigenetic effects of childhood trauma. In this regard it is noteworthy that a recent postmortem study has reported a significant effect of childhood abuse on the epigenetic regulation of glucocorticoid receptors in suicide victims (McGowan et al, 2009). Such a mechanism might also explain why Binder et al (2008) found a $G \times E$ effect on PTSD symptoms with childhood trauma but not trauma exposure in adulthood.

The strengths of this study include the fact that we studied an African-American sample similar to that of Binder et al (2008), and that we corrected for population stratification in this admixed population using ethnic factor scores derived from 186 AIMs. It turned out that correction for population stratification was necessary as European admixture significantly influenced the risk of suicide attempt. There are a few limitations to our study. The childhood trauma data were derived from a self-report questionnaire. Nevertheless, the CTQ has been shown to have high reliability and validity (Bernstein et al, 1997, 1994; Bernstein and Fink, 1998; Scher et al, 2001; Thombs et al, 2007). Studies have shown that the retrospective reporting of childhood trauma is valid (Bifulco et al, 1997; Goodman et al, 1999) and is not influenced by psychiatric state at the time of report (Fergusson et al, 2000). In this regard it is noteworthy that all the patients were abstinent at the time of study. Most patients were interviewed in the last week of a 3-week stay in a locked substance abuse residential program. Other limitations are that the CTQ does not include an exhaustive list of potentially traumatic events that are commonly experienced in youth and that adult exposure to potentially traumatic events was not assessed. Because men predominated in the sample, it was not possible to determine potential sex differences. Moreover, the nature of each suicide attempt was not 
systematically recorded. As the complete SCID was not administered, data about possible comorbid disorders such as PTSD and depression were not available. Finally, the sample size decreased to 474 (112 suicide attempters) when examining gene $\times$ environment interactions although this sample size is comparable to that for the significant findings in Binder et al (2008). Our results should therefore be interpreted with caution until replicated by others.

In conclusion, this study reports that significant interaction between childhood trauma and the HPA axis gene FKBP5 appears to raise the risk of attempting suicide. However, as only up to $19 \%$ of the variance in risk for a suicide attempt was accounted for, and as the sample was African American and the suicide attempts were largely confined to the substance-dependent patients, it is not known whether the results of our study can be extrapolated to other groups and nonsubstance-dependent patients. Therefore, the generalizability of the results is an issue and further studies are indicated in relation to suicidal behavior in other diagnostic and ethnic groups.

\section{DISCLOSURE}

The authors declare no conflict of interest.

\section{REFERENCES}

Afifi T, Enns M, Cox B, Asmundson G, Stein M, Sareen J (2008). Population attributable fractions of psychiatric disorders and suicide ideation and attempts associated with adverse childhood experiences. Am J Pub Health 98: 946-952.

Arato M, Banki C, Bissette G, Nemeroff C (1989). Elevated CSF CRH in suicide victims. Biol Psychiatry 25: 255-259.

Arnow B (2004). Relationships between childhood maltreatment, adult health and psychiatric outcomes, and medical utilization. J Clin Psychiatry 65(Suppl 12): 10-15.

Beatrais A, Joyce P, Mulder R (1997). Precipitating factors and life events in serious suicide attempters among youth. J Am Acad Child Adolesc Psychiatry 36: 1543-1551.

Bernstien D, Ahluvalia T, Handelsman L (1997). Validity of the Childhood Trauma Questionnaire in an adolescent psychiatric population. J Am Acad Child Adolesc Psychiatry 36: 340-348.

Bernstien D, Fink L, Handelsman L, Foote J, Lovejoy M, Wenzel K et al (1994). Initial reliability and validity of a new retrospective measure of child abuse and neglect. Am J Psychiatry 151: 1132-1136.

Bernstein D, Fink L (1998). Childhood Trauma Questionnaire: A Retrospective Self-Report Manual. The Psychological Corporation: San Antonio, TX.

Bernstein DP, Stein JA, Newcomb MD, Walker E, Pogge D, Ahluvalia $\mathrm{T}$ et al (2003). Development and validation of a brief screening version of the Childhood Trauma Questionnaire. Child Abuse Negl 27: 169-190.

Bifulco A, Brown G, Lillie A, Jarvis J (1997). Memories of childhood neglect and abuse: corroboration in a series of sisters. J Child Adol Psychiatry 38: 365-374.

Binder EB (2009). The role of FKBP5, a co-chaperone of the glucocorticoid receptor in the pathogenesis and therapy of affective and anxiety disorders. Psychoneuroendocrinology 34(Suppl 1): S186-S195.

Binder EB, Salyakina D, Lichtner P, Wochnik GM, Ising M, Pütz B et al (2004). Polymorphisms in FKBP5 are associated with increased recurrence of depressive episodes and rapid response to antidepressant treatment. Nat Genet 36: 1319-1325.
Binder EB, Bradley RG, Liu W, Epstein MP, Deveau TC, Mercer KB et al (2008). Association of FKBP5 polymorphisms and childhood abuse with risk of posttraumatic stress disorder symptoms in adults. JAMA 299: 1291-1305.

Bondy B, Buettner A, Zill P (2006). Genetics of suicide. Mol Psychiatry 11: 336-351.

Brodsky BS, Stanley B (2007). Adverse childhood experiences and suicidal behavior. Psychiatr Clin North Am 31: 223-235.

Bunney W, Fawcett J (1965). Possibility of a biochemical test for suicidal potential. Arch Gen Psychiatry 17: 317-332.

Bunney W, Fawcett J, Davis J, Gifford S (1969). Further evaluation of urinary 17-hydroxycortocosteroids in suicidal patients. Arch Gen Psychiatry 21: 138-150.

Castle K, Duberstein P, Meldrum S, Conner K, Conwell Y (2004). Risk factors for suicide in blacks and whites; an analysis of data from the 1993 National Mortality Follow back Survey. Am J Psychiatry 161: 452-458.

Coryell W, Schlesser M (2001). The dexamethasone suppression test and suicide prediction. Am J Psychiatry 158: 748-753.

Crane C, Williams J, Hawton K, Arensman E, Hjelmeland $\mathrm{H}$, Bille-Brahe $U$ et al (2007). The association between life events and suicide intent in self-poisoners with and without a history of self-harm: a preliminary study. Suicide Life Threat Behav 37: 367-378.

Davidson J, Hughes D, George L, Blazer D (1996). The association of sexual assault and attempted suicide in the community. Arch Gen Psychiatry 53: 550-555.

De Bellis MD, Chrousos GP, Dorn LD, Burke L, Helmers K, Kling MA et al (1994). Hypothalamic-pituitary-adrenal axis dysregulation in sexually abused girls. J Clin Endocrinol Metab 78: 249-255.

Fergusson D, Horwood L, Woodward L (2000). The stability of child abuse reports: a longitudinal study of the reporting behaviour of young adults. Psychol Med 30: 529-544.

Goodman LA, Thompson KM, Weinfurt K, Corl S, Acker P, Mueser KT et al (1999). Reliability of reports of violent victimization and posttraumatic stress disorder among men and women with serious mental illness. J Trauma Stress 12: 587-599.

Harris E, Barraclough B (1997). Suicide as an outcome for mental disorders. A meta-analysis. Br J Psychiatry 170: 205-228.

Heikkinen M, Aro H, Lonnqvist J (2007). Recent life events, social support and suicide. Acta Psychiatr Scand 89: 65-67.

Heim C, Plotsky P, Nemeroff C (2004). Importance of studying the contribution of early adverse experiences to neurobiological finding in depression. Neuropsychopharmacology 29: 641-648.

Heim C, Nemeroff CB (2009). Neurobiology of posttraumatic stress disorder. CNS Spectr 14(1 Suppl 1): 13-24.

Hodgkinson CA, Yuan Q, Xu K, Shen PH, Heinz E, Lobos EA et al (2008). Addictions biology: haplotype-based analysis for 130 candidate genes on a single array. Alcohol Alcohol 43: 505-515.

Holmes W, Slap G (1998). Sexual abuse of boys: definition, prevalence, correlates, sequelae, and management. JAMA 280: 1855-1862.

Hyman S, Garcia M, Sinha R (2006). Gender specific associations between types of childhood maltreatment and the onset, escalation and severity of substance use in cocaine dependent adults. Am J Drug Alcohol Abuse 32: 655-664.

Ising M, Depping AM, Siebertz A, Lucae S, Unschuld PG, Kloiber S et al (2008). Polymorphisms in the FKBP5 gene region modulate recovery from psychosocial stress in healthy controls. Eur $J$ Neurosci 28: 389-398.

Johnsson E, Fridell M (1997). Suicide attempts in a cohort of drug abusers: a 5-year follow-up study. Acta Psychiatr Scand 96: 362-366.

Kosten T, Rounsaville B (1988). Suicidality among opioid addicts: 2.5 year follow up. Am J Drug Alcohol Abuse 14: 357-369.

Lindqvist D, Isaksson A, Träskman-Bendz L (2008). Salivary cortisol and suicidal behavior-a follow up study. Psychoneuroendocrinology 33: 1061-1068. 
MaMillan H, Fleming J, Trocme N, Boyle M, Wong M, Racine Y et al (1997). Prevalence of child physical and sexual abuse in the community. Results from the Ontario Health Supplement. JAMA 278: 131-135.

McGowan P, Sasaki A, D’Alessio C, Dymov S, Labonte B, Szyf M et al (2009). Epigenetic regulation of the glucocorticoid receptor in human brain associates with childhood abuse. Nat Neurosci 12: $342-348$

Meaney M. (2001). Maternal care, gene expression, and the transmission of individual differences in stress reactivity across generations. Annu Rev Neurosci 24: 1161-1192.

Molnar B, Berkman L, Buka S (2001). Psychopathology, childhood sexual abuse, and other childhood adversities: relative links to subsequent suicidal behavior in the US. Psychol Med 31: 963-977.

Moscicki E, Carroll P, Rae D, Locke B, Roy A, Regier D (1998). Suicide attempts in the Epidemiologic Catchment Area Study. Yale J Biol Med 61: 259-268.

Nelson E, Health A, Madden P, Cooper L, Dinwiddle S, Bucholz K et al (2002). Association between self-reported childhood sexual abuse and adverse psychosocial outcomes. Results from a twin study. Arch Gen Psychiatry 59: 139-145.

Nelson EC, Heath AC, Lynskey MT, Bucholz KK, Madden PA, Statham DJ et al (2006). Childhood sexual abuse and risks for licit and illicit drug-related outcomes: a twin study. Psychol Med 36: 1473-1483.

Nemeroff C. (2004). Neurobiological consequences of childhood trauma. J Clin Psychiatry 65: 18-28.

Nemeroff CB, Owens MJ, Bissette G, Andorn AC, Stanley M (1988). Reduced corticotrophin releasing factor binding sites in the frontal cortex of suicide victims. Arch Gen Psychiatry 45: 577-579.

Pfennig A, Kunzel H, Kern N, Ising M, Majer M, Fuchs B et al (2005). Hypothalamus-pituitary-adrenal system regulation and suicidal behavior in depression. Biol Psychiatry 57: 336-342.

Osvath P, Voros V, Fekete S (2004). Life events and psychopatholgy in a group of suicide attempters. Psychopathology 37: 36-40.

Paykel G, Prusoff B, Myers J (1975). Suicide attempts and recent life events: a controlled comparison. Arch Gen Psychiatry 32: 327-333.

Richardson HN, Lee SY, O’Dell LE, Koob GF, Rivier CL (2008). Alcohol self-administration acutely stimulates the hypothalamic-pituitary-adrenal axis, but alcohol dependence leads to a dampened neuroendocrine state. Eur J Neurosci 28: 1641-1653.

Roy A (1992). Hypothalamic-pituitary-adrenal axis function and suicidal behavior in depression. Biol Psychiatry 32: 812-816.

Roy A (2001a). Childhood trauma and attempted suicide in alcoholics. J Nerv Ment Disease 189: 120-121.

Roy A (2001b). Characteristics of cocaine dependent patients who attempt suicide. Am J Psychiatry 158: 1214-1219.

Roy A (2002a). Urinary-free cortisol and childhood trauma in cocaine dependent adults. J Psychiatr Res 36: 173-177.
Roy A (2002b). Characteristics of opiate dependent patients who attempt suicide. J Clin Psychiatry 63: 403-407.

Roy A (2003). Characteristics of drug addicts who attempt suicide. Psychiatr Res 121: 99-103.

Roy A (2005a). Reported childhood trauma and suicide attempts in schizophrenic patients. Suicide Life Threat Behav 35: 690-693.

Roy A (2005b). Childhood trauma and suicidal behavior in male cocaine dependent patients. Suicide Life Threat Behav 31: 194-196.

Roy A, Agren H, Pickar D, Linnoila M, Doran A, Cutler N et al. (1986). Reduced CSF concentrations of homovanillic acid and homovanillic acid to 5-hydroyindoleacetic acid ratios in depressed patients: relationship to suicidal behavior and dexamethasone nonsuppression. Am J Psychiatry 143: $1539-1545$

Roy A, Hu X-Z, Janal M, Goldman D (2007). Interaction between childhood trauma and serotonin gene variation in attempting suicide. Neuropsychopharmacology 32: 2046-2052.

Roy A, Janal M (2007). Risk factors for suicide attempts among alcoholics. Arch Suicide Res 11: 211-217.

Sánchez M, Noble P, Lyon C, Plotsky P, Davis M, Nemeroff C et al. (2005). Alterations in diurnal cortisol rhythm and acoustic startle response in nonhuman primates with adverse rearing. Biol Psychiatry 57: 373-381.

Sarchiapone M, Carli V, Cuomo C, Roy A (2007). Childhood trauma and suicide attempts in unipolar depressed patients. Depress Anxiety 24: 268-272.

Scher CD, Stein MB, Asmundson GJ, McCreary DR, Forde DR (2001). The childhood trauma questionnaire in a community sample: psychometric properties and normative data. J Trauma Stress 14: 843-857.

Spitzer RL, Williams JBW, Gibbon M (1995). Structured Clinical Interview for DSM-IV (SCID). New York State Psychiatric Institute, Biometrics Research: New York.

Stephens M, Donnelly P (2003). A comparison of Bayesian methods for haplotype reconstruction from population genotype data. Am J Hum Genet 73: 1162-1169.

Thombs BD, Lewis C, Bernstein DP, Medrano MA, Hatch JP (2007). An evaluation of the measurement equivalence of the Childhood Trauma Questionnaire - short form across gender and race in a sample of drug-abusing adults. J Psychosom Res 63: 391-398.

Weaver IC, Champagne FA, Brown SE, Dymov S, Sharma S, Meaney MJ et al. (2005). Reversal of maternal programming of stress responses in adult offspring through methyl supplementation: altering epigenetic marking later in life. J Neurosci 25: 11045-11054.

Wilcox H, Storr C, Breslau N (2009). Posttraumatic stress disorder and suicide attempts in a sample of urban American young adults. Arch Gen Psychiatry 66: 305-311.

Willour VL, Chen H, Toolan J, Belmonte P, Cutler DJ, Goes FS et al. (2009). Family-based association of FKBP5 in bipolar disorder. Mol Psychiatry 14: 261-268. 